\title{
RESULTS FROM THE UCLA/FNPL UNDERDENSE PLASMA LENS EXPERIMENT
}

\author{
M. C. THOMPSON*, H. BADAKOV, J. B. ROSENZWEIG, G. TRAVISH ${ }^{\dagger}$ \\ Department of Physics and Astronomy, \\ University of California, Los Angeles \\ Los Angeles, CA 90095 \\ E-mail: thompson93@llnl.gov
}

H. EDWARDS, R. FLILLER, G. M. KAZAKEVICH, P. PIOT, J. SANTUCCI

Fermi National Accelerator Laboratory,

Batavia, Illinois, 60510

J. LI AND R. TIKHOPLAV

University of Rochester,

Rochester, New York 14627

\begin{abstract}
A gaussian underdense plasma lens with peak density $5 \times 10^{12} \mathrm{~cm}^{-3}$ and a full width half maximum (FWHM) length of $2.2 \mathrm{~cm}$ has been used to focus a relativistic electron beam. This plasma lens is equivalent in strength to a quadrupole magnet with a $150 \mathrm{~T} / \mathrm{m}$ field gradient. The lens focused a $15 \mathrm{MeV}, 16 \mathrm{nC}$ electron beam with initial dimensions $\sigma_{x, y} \approx 650 \mu \mathrm{m}$ and $\sigma_{z} \approx 6.5 \mathrm{~mm}$ onto an optical transition radiation (OTR) screen $\sim 2 \mathrm{~cm}$ downstream of the lens. The average transverse area of the plasma focused electron beam was typically demagnified by a factor of 23. The evolution of the beam envelope in the area near the beam waist was measured for both round beams and asymmetric beams with $\mathrm{x}$ :y aspect ratios as large as 1:5. The light from the OTR screen in the round beam case was also imaged into a streak camera in order to directly measure the correlation between $\mathrm{z}$ and $\sigma_{r}$ within the beam.
\end{abstract}

\section{INTRODUCTION}

Plasma lenses are of great interest to the accelerator physics community because they can theoretically provide radially symmetric focusing gradi-

${ }^{*}$ Current Affiliation: Lawrence Livermore National Laboratory
${ }^{\dagger}$ Spokesman for the collaboration at Erice. 
ents on the order of $10 \mathrm{MT} / \mathrm{m}$, which exceeds the strength of conventional quadrupole lens by several orders of magnitude ${ }^{1-3}$. Increasing focusing gradients to this level would greatly aid in achieving the small spot sizes and high luminosity necessary for future $e^{+} e^{-}$colliders such as the planned International Linear Collider (ILC). Additionally, it has been shown that adiabatic plasma lenses can overcome the synchrotron-radiation limit on final focus spot size ${ }^{4}$. The underdense regime of plasma lens operation, in which the density of beam electrons $n_{b}$ is greater than half of the plasma density $n_{p}$, is considered the most advantageous for the focusing of electron beams in many scenarios ${ }^{2,4}$.

The most commonly considered regimes of plasma lens operation are the overdense $\left(n_{b}<<n_{p}\right)$ and underdense $\left(n_{b}>n_{p} / 2\right)$ regimes ${ }^{3}$. In the overdense case the plasma essentially cancels the beam space charge and allows it to focus under its magnetic self forces. Since the beam magnetic self forces are generally not uniform over the length of the bunch or linear over its breadth, overdense lenses typically have significant abberations. In the underdense case the strong space charge of the electron beam ejects the plasma electrons entirely, leaving a uniform ion column. It can be shown that the radial focusing force of this ion column is given by

$$
F_{\text {radial }}=-2 \pi n_{p} e^{2} r
$$

where $e$ is the electron charge and $r$ is the radial distance from the axis. An underdense plasma lens is hence free of spherical aberrations in the ideal limit, and is equivalent in strength to a quadrupole focusing magnet with a magnetic gradient of $\left(3 \times 10^{-11}\right) n_{p} \mathrm{~T} / \mathrm{m}$. A plasma lens with the relatively modest density of $5 \times 10^{12} \mathrm{~cm}^{-3}$ therefore has the strength of a $150 \mathrm{~T} / \mathrm{m}$ quadrupole. State-of-the-art superconducting quadrupoles, by comparison, have maximum gradients on the order of $250 \mathrm{~T} / \mathrm{m}$. Plasma lenses also focus both transverse beam axes simultaneously. The drawback of plasma lenses in either regime of operation is that only about $80 \%$ of the beam is focused. Due to the finite time it takes for the plasma to react to the beam space charge, the head of the beam is not subject to the plasma focusing forces.

The focal length $f$ of a thin plasma lens is given by $f=1 / K l$, where $K$ is the lens focusing strength and $l$ is the lens length. For an underdense plasma lens

$$
\frac{1}{f}=K l=\int \frac{2 \pi r_{e} n_{p}(l)}{\gamma} d l,
$$

where $r_{e}$ is the classical electron radius, $\gamma$ is the Lorentz factor, and $n_{p}(l)$ is the function that describes the variation in plasma density along the 
electron beam's path through the lens.

\section{EXPERIMENTAL METHODOLOGY}

Our underdense plasma lens experiment, which evolved from earlier proposals ${ }^{5,6}$ and follows previous electron plasma lens work in other regimes 7-10, was conducted at the Fermilab NICADD Photoinjector Laboratory (FNPL) at FNAL. A schematic of the experiment is shown in Fig. 1. A bulk

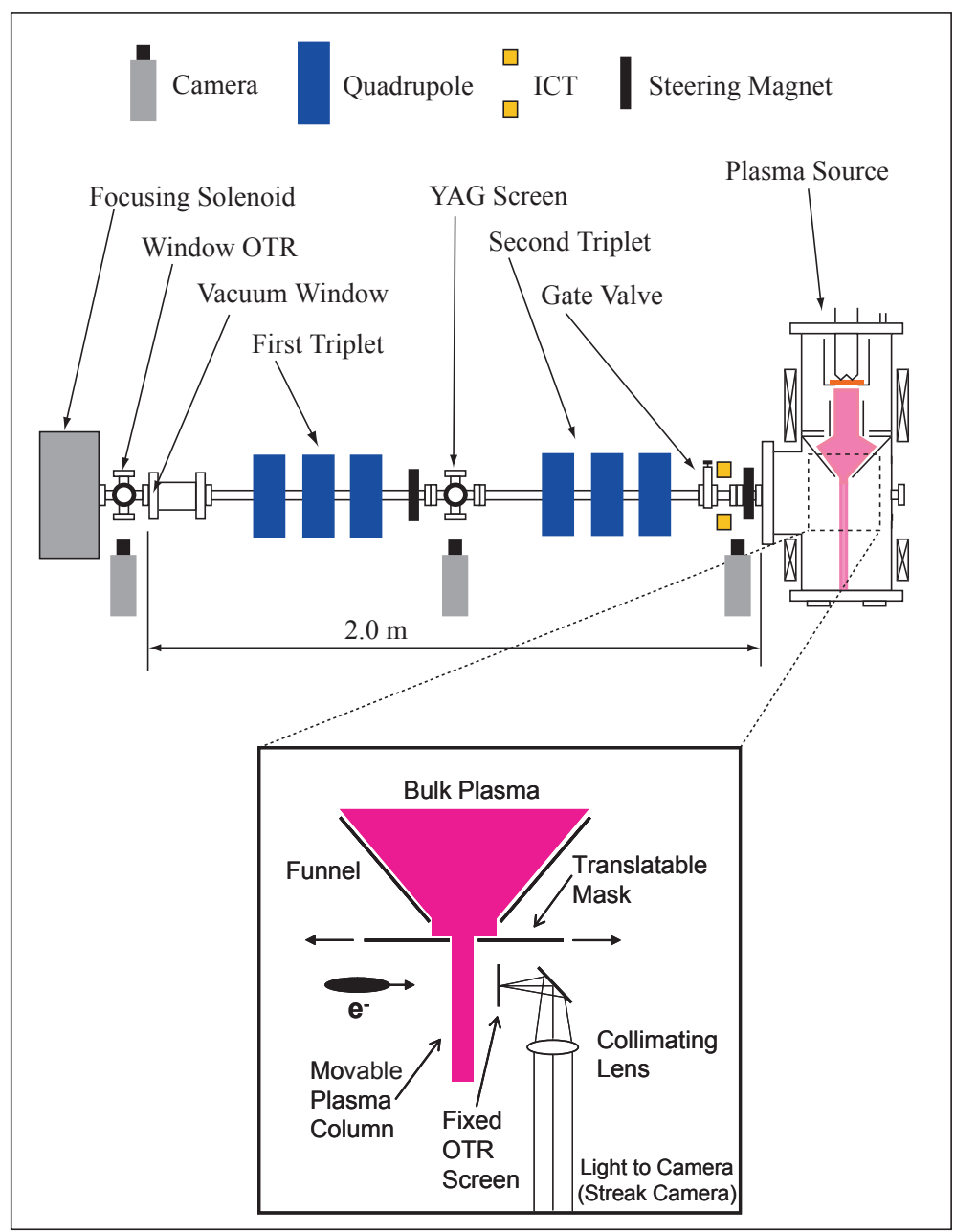

Figure 1. Schematic of the plasma lens experiment beamline and apparatus. 
Table 1. Experimental Parameters

\begin{tabular}{|l|c|}
\hline Peak Plasma Density & $4.7 \times 10^{12} \mathrm{~cm}^{-3}$ \\
Plasma Thickness $\left(\sigma_{p}\right)$ & $9.9 \mathrm{~mm}$ \\
Beam Charge & $16 \mathrm{nC}$ \\
Beam Duration $\left(\sigma_{t}\right)$ & $22 \mathrm{ps}$ \\
Initial Beam Radius $\left(\sigma_{r}\right)$ & $950 \mu \mathrm{m}$ \\
Beam Emittance $\left(\epsilon_{n}\right)$ & $90 \mathrm{~mm}-\mathrm{mrad}$ \\
Peak Beam Density & $2.2 \times 10^{12} \mathrm{~cm}^{-3}$ \\
\hline
\end{tabular}

column of plasma approximately $5 \mathrm{~cm}$ FWHM is produced using our pulsed argon discharge plasma source ${ }^{11}$. The gaussian plasma lens is formed by selecting a slice of this static plasma column with a translatable mask with a $1.25 \mathrm{~cm}$ wide slit. This arrangement allows the distance between the plasma lens column and the fixed OTR diagnostic screen to be varied without altering the OTR collection optics. Measurements have shown that the plasma lens column properties, listed in Table 1, vary only slightly as the mask is translated through the $1 \mathrm{~cm}$ region of interest. An in-vacuum, 160 $\mathrm{mm}$ focal length, 2 inch diameter lens provides point-to-parallel collimation of the OTR produced when the electron beam emerges from the back of a polished aluminum foil. The OTR is then transported either $30 \mathrm{~cm}$ to a conventional CCD camera with a $75 \mathrm{~mm}$ compound camera lens or $7 \mathrm{~m}$ to a streak camera where the light is imaged onto the streak slit using a single $280 \mathrm{~mm}$ focal length lens.

The electron beam for the experiment is provided by the FNPL electron accelerator. The FNPL accelerator is a $15 \mathrm{MeV}$ electron linac ${ }^{12}$ consisting of a normal conducting L-band RF gun with a cesium telluride photo-cathode and a 9-cell superconducting accelerating cavity. After acceleration, the electron beam is propagated at a tight focus through a $10 \mu \mathrm{m}$ thick aluminum vacuum foil, used to isolate the plasma experiment vacuum from the rest of the beam line, and refocused into the plasma lens experiment, Fig. 1.

The plasma lens experiment parameters are listed in Table 1. Note that $n_{\text {b,peak }} \approx n_{\text {p,peak }} / 2$, which puts the experiment just on the boundary of the underdense regime at the entrance to the plasma lens. This is a lower bound, however, since the beam is substantially denser compared to the edge of the gaussian plasma column which it first encounters and, since the lens is relatively thick, the beam core compresses somewhat by the time it reaches the center of the plasma lens. It is therefore clear that the beam focusing is governed by underdense dynamics at the threshold of the regime. Given the values in Table 1, Eq. 2 predicts $f=1.5 \mathrm{~cm}$ for the gaussian plasma lens. The observed focal length was $f \cong 1.9 \mathrm{~cm}$. 
The agreement between these two values is reasonable considering that the gaussian plasma lens does not satisfy the thin lens criteria $(f>>l)$, which is the limit assumed in Eq. 2.

\section{UNDERDENSE PLASMA FOCUSING}

Three distinct series of measurements were made during this experiment: time integrated measurements of round focused spots, time resolved measurements of round focused spots, and time integrated measurements of focused beams with ratios of $\mathrm{x}$ dimension to $\mathrm{y}$ dimension of up to about 1:5. Each of these measurements provides information on a different aspect of the underdense plasma lens performance and behavior.

\subsection{Time Integrated Measurements of Round Beam Focusing and the Focused Beam Envelope}

The focusing effect of our underdense plasma lens was strong and highly repeatable. Figs. 2 and 3 show the effect of plasma focusing on the electron beam spot size as observed with the conventional CCD camera. Note that the focused beam is imaged at the lens focus, $f=1.9 \mathrm{~cm}$, and shows only the core of the beam since our camera lacked sufficient dynamic range to simultaneously record the unfocused portion of the beam along with the focused core. The unfocused beam is nearly round with $\mathrm{x}$ FWHM $=1630$ $\mu \mathrm{m}$ and y FWHM $=1540 \mu \mathrm{m}$. The underdense plasma lens focuses this beam down to $\mathrm{x} F W H M=260 \mu \mathrm{m}$ and $\mathrm{y} F W H M=420 \mu \mathrm{m}$ which is a

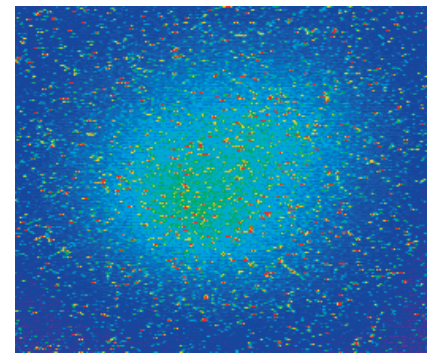

Unfocused - 5 electron pulses

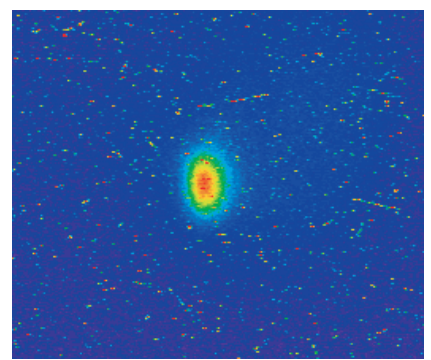

Focused - 1 electron pulse

Figure 2. False color images of the unfocused (left) and focused (right) electron beam spots as observed on the OTR screen after the plasma lens. The unfocused image is shown at five times normal intensity in order to provide sufficient image contrast. Both images use the same scaled-to-intensity color map. 

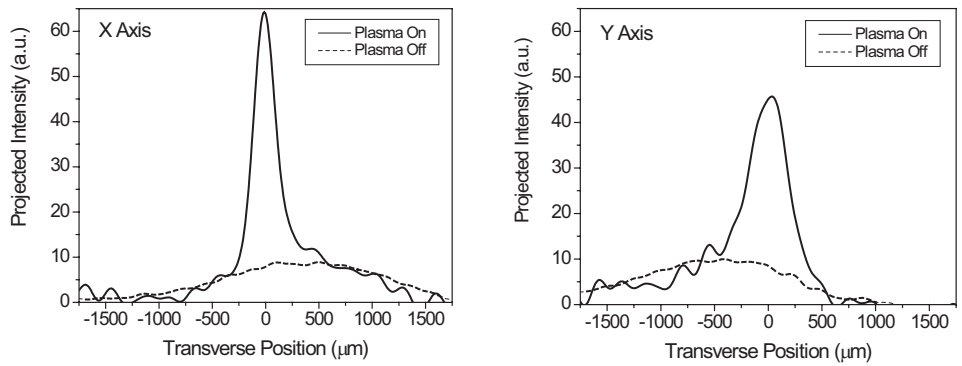

Figure 3. Plots of the image intensity of the beam images shown in Fig. 2 (normalized to 1 electron pulse) in the $\mathrm{x}$ axis (left) and $\mathrm{y}$ axis (right).

demagnification of 6.3 in $\mathrm{x}$ and 3.66 in $\mathrm{y}$. The average transverse area of the beam is consequently reduced by a factor of 23 . This area reduction equates to a boost in the core beam density from $2.2 \times 10^{12} \mathrm{~cm}^{-3}$ in the unfocused case to $5 \times 10^{13} \mathrm{~cm}^{-3}$ in the focused case.

Measurements of the focused beam envelope were made by taking spot size measurements at various lens / OTR screen spacings, Fig. 4. The variation of the beam spot size near the focus should be describable (neglecting space charge effects) by the beam envelope equation:

$$
\frac{d^{2} \sigma_{x}}{d z^{2}}+K \sigma_{x}=\frac{\epsilon_{x}^{2}}{\sigma_{x}^{3}},
$$

where $\sigma_{x}$ is the rms beam size in $x, \epsilon_{x}$ is the geometric beam emittance in $x$, and $K$ is once again the focusing strength. The equivalent equation for the other transverse direction can be written by substituting $y$ for $x$. We are

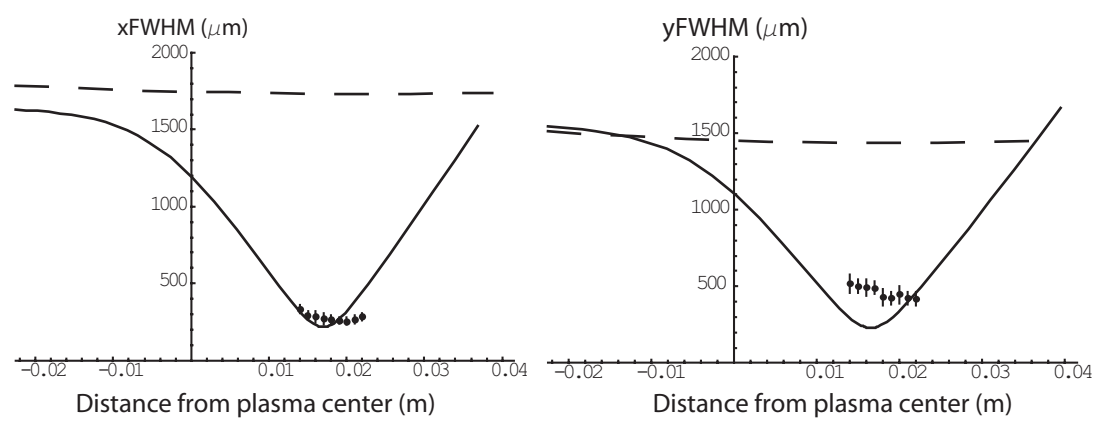

Figure 4. Measurements of underdense plasma lens focusing in the $\mathrm{x}$ axis (left) and $\mathrm{y}$ axis (right). Preliminary solution of the envelope equation are also shown. The upper dashed lines are solutions to the beam line transport model, while the lower solid lines are solutions to the lens model. 
using Eq. 3 to model both the transport of the beam from the vacuum foil to the plasma lens, and the effect of the plasma lens itself. The results of our preliminary modeling efforts clearly illustrate the strong focusing effect of the plasma lens and are shown, along with the beam envelope data, in Fig. 4. We should be able to refine the rough agreement between the data and the solutions to Eq. 3 as the data analysis progresses. It should also be possible to extract quantitative information about the plasma lens' aberrations from the beam envelope analysis.

\subsection{Time Resolved Measurements of Focusing}

A series of time resolved measurements of the plasma focusing were made by imaging the beam OTR light onto the slit of a Hamamatsu series C5680 streak camera. An example of one the images recorded on the streak camera, along with an analysis of that shot, is shown in Fig. 5. As expected, the intensity profile of the focused beam in the time domain remains roughly gaussian while the beam is radially larger at the head than in the middle or tail. Width measurements were made by examining 5 ps wide swaths of the beam. The time resolution of the streak camera had previously been measured to be below 2 ps. While the time domain behavior of the plasma focusing is in general agreement with theoretical predictions, the raw data does not have as large a change between head and tail diameter as expected from either theory or the time integrated measurements. This apparent lack of transverse resolution seems to originate from limitations in the performance of our OTR light transport and may result from slight optics misalignments, the large angles inherent in such a strongly focused
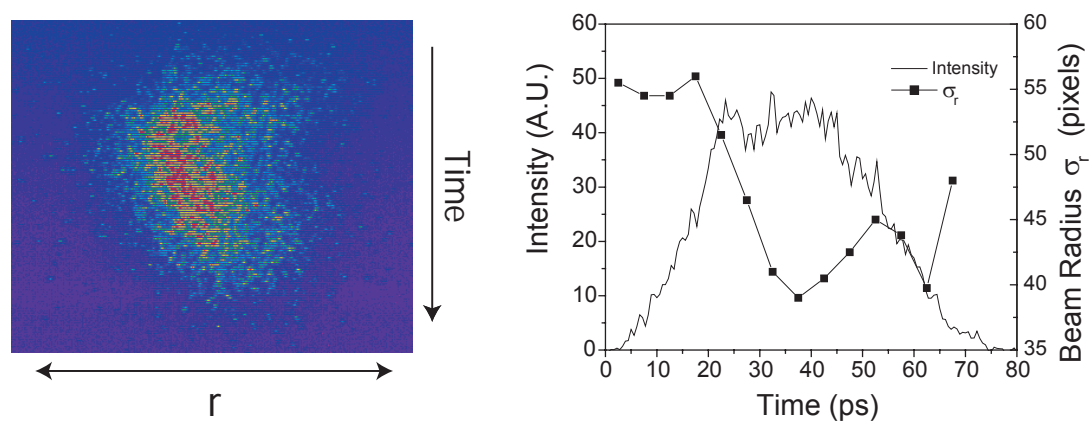

Figure 5. A false color streak camera image of the plasma focused beam (left) with initial analysis of the image (right). 


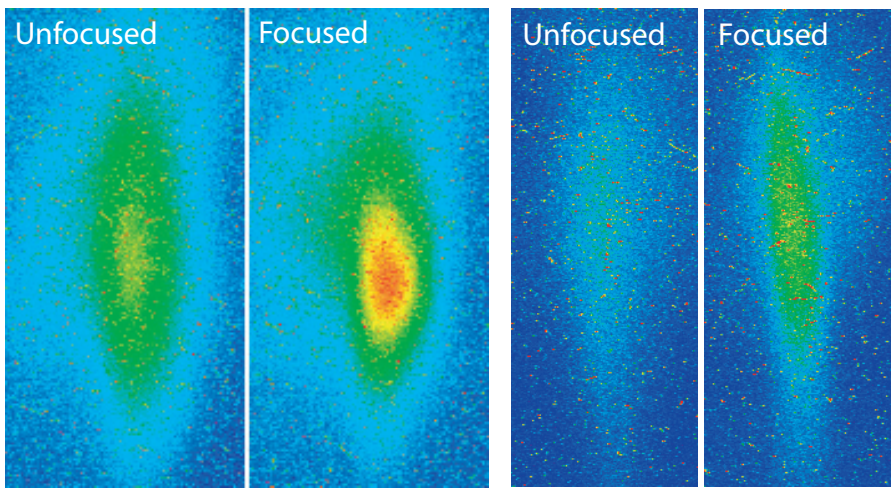

Figure 6. False color images of a beams with an initial aspect ratio of 1:3.4 (at left) and 1:4.5 (at right). In each set, both images show the same number of beam pulses.

beam, and other effects. Analysis of the streak camera data is ongoing.

\subsection{Elliptical Beam Focusing}

Our studies of underdense plasma lens focusing also included an examination of elliptical beam focusing. Fig. 6 shows examples of time integrated measurements of elliptical beams with 1:3.4 and 1:4.5 aspect ratios between the $\mathrm{x}$ and $\mathrm{y}$ dimensions. A clear plasma focusing effect is visible in both cases. The highest aspect ratio examined was 1:4.5, largely because it was impractical to transport flat beams with large asymmetric emittances to the experiment. We plan to make a quantitative analysis of the elliptical beam data in a manner similar to round beam analysis discussed above.

The motivation behind these measurements of modestly asymmetric beams is the desire to understand how the dynamics of underdense plasma focusing change as the beam aspect ratio increases. The development of such an understanding is vital if underdense lenses are to be applied in a ILC context where aspect beam ratios at the interaction point are 1:100. Our measurements are a start, but future experiments with flat, very high aspect ratio beams will be needed.

\section{Conclusions}

We have measured extreme demagnification of a high brightness electron beam by a strong underdense plasma lens. The collected data include both time integrated and time resolved measurements of the focused beam spot and its envelope. While data reduction and analysis are ongoing, the 
general behavior of the plasma lens matches our theoretical expectations very well. A full analysis of this underdense plasma lens experiment will be reported elsewhere ${ }^{13}$.

\section{ACKNOWLEDGEMENTS}

This work was performed under the auspices of the US Department of Energy under Contract No. DE-FG03-92ER40693 and W-7405-ENG-48.

\section{References}

1. P. Chen. Part. Accel., 20, 171 (1987).

2. P. Chen, et al., Phys. Rev. D, 40, 923 (1989).

3. J. Su, et al., Phys. Rev. A, 41, 3321 (1990).

4. P. Chen, et al., Phys. Rev. Lett., 64, 1231 (1990).

5. H. Suk, et al., In Proc. PAC 1999, 3708 (1999).

6. M. C. Thompson, et al., In Proc. PAC 2001, 4014 (2001).

7. H. Nakanishi, et al., Phys. Rev. Lett., 66, 1870 (1991).

8. G. Hairapetian, et al., Phys. Rev. Lett., 72, 2403 (1994).

9. R. Govil, et al., Phys. Rev. Lett., 83, 3202 (1999).

10. P. R. Bolton, et al., Int. J. Mod. Phys. A 18, 2843 (2003).

11. M. C. Thompson, et al., Rev. Sci. Instr., 76, 013303 (2005).

12. J.-P. Carneiro, et al., In Proc. PAC 1999, 2027 (1999).

13. M. C. Thompson, et al., Phys. Rev. Lett., (to be submitted). 